\title{
Mapping Surface Hydrophobicity of $\alpha$-Synuclein Oligomers at the Nanoscale
}

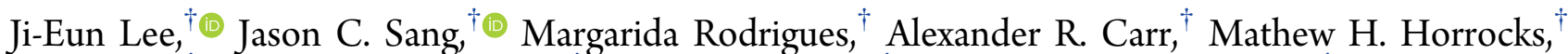
Suman De, ${ }^{\dagger}$ Marie N. Bongiovanni, ${ }^{\dagger}$ Patrick Flagmeier, ${ }^{\dagger}{ }^{\oplus}$ Christopher M. Dobson, ${ }^{\dagger}$ David J. Wales, ${ }^{\dagger}$ Steven F. Lee, ${ }^{* \dagger}$ and David Klenerman ${ }^{*},+$,

${ }^{\dagger}$ Department of Chemistry, University of Cambridge, Lensfield Road, Cambridge CB2 1EW, United Kingdom

${ }^{\ddagger}$ UK Dementia Research Institute, University of Cambridge, Cambridge CB2 OXY, United Kingdom

Supporting Information
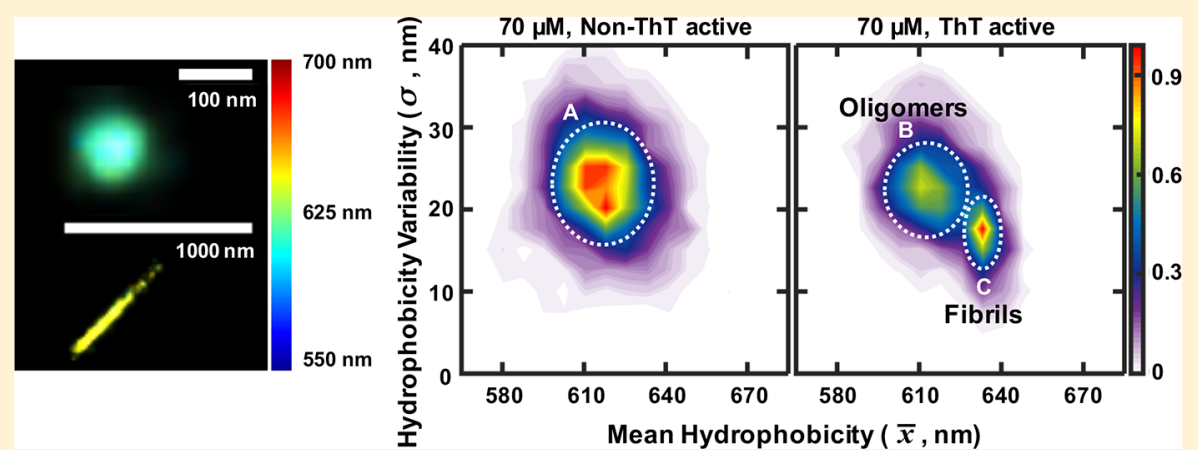

ABSTRACT: Proteins fold into a single structural ensemble but can also misfold into many diverse structures including small aggregates and fibrils, which differ in their toxicity. The aggregate surface properties play an important role in how they interact with the plasma membrane and cellular organelles, potentially inducing cellular toxicity, however, these properties have not been measured to date due to the lack of suitable methods. Here, we used a spectrally resolved, super-resolution imaging method combined with an environmentally sensitive fluorescent dye to measure the surface hydrophobicity of individual aggregates formed by the protein $\alpha$-synuclein $(\alpha \mathrm{S})$, whose aggregation is associated with Parkinson's disease. We show that the surface of soluble oligomers is more hydrophobic than fibrils and populates a diverse range of coexisting states. Overall, our data show that the conversion of oligomers to fibril-like aggregates and ultimately to fibrils results in a reduction in both hydrophobicity and the variation in hydrophobicity. This funneling characteristic of the energy landscape explains many of the observed properties of $\alpha \mathrm{S}$ aggregates and may be a common feature of aggregating proteins.

KEYWORDS: Super-resolution spectroscopy, spectral imaging, Nile red, hydrophobicity, protein aggregation, alpha-synuclein

$\mathrm{T}$ he aggregation of monomeric proteins into small soluble oligomers, and ultimately insoluble $\beta$-sheet-rich fibrils, plays a key role in several neurodegenerative disorders, including Alzheimer's, Parkinson's, and prion diseases. A number of studies have shown that small aggregates present in solution, oligomers, are the main cytotoxic species, ${ }^{1,2}$ while fibrils are capable of seeding aggregation and spreading from cell to cell in the brain. ${ }^{3}$ There have been significant advances in understanding the structure of fibrils of amyloid- $\beta(\mathrm{A} \beta)$ and $\alpha$-synuclein $(\alpha \mathrm{S})$ in vitro ${ }^{4,5}$ and in cells, ${ }^{6-8}$ and measurement by atomic force microscopy and electron microscopy of the aggregates formed during this process. ${ }^{9-11}$ However, very little is known about the surface properties of these aggregates despite the fact that surface properties will determine how aggregates interact with cell membranes and cellular components and hence may play a key role in the mechanism by which aggregates are toxic to cells. In particular, the surface hydrophobicity of the protein aggregates associated with the neurodegenerative diseases is thought to be central to their toxicity, ${ }^{12}$ since more hydrophobic aggregates will bind more strongly to membranes and cellular organelles.

We have previously studied the aggregation of $\alpha \mathrm{S}$, a major constituent of Lewy bodies and the pathological hallmark of Parkinson's disease, ${ }^{13}$ covalently coupled a donor or acceptor fluorophore using single-molecule Förster resonance energy transfer (FRET) measurements and kinetic modeling. ${ }^{1,14}$ We identified three species: (1) low FRET oligomers, which were easily degradable by proteinase $\mathrm{K}(\mathrm{PK})$, and convert into (2) high FRET, PK resistant and more toxic oligomers, which then convert to (3) fibril-like high FRET oligomers that grow into fibrils. However, these experiments did not provide any information on the surface properties of aggregates. More recently we developed a multidimensional super-resolution

Received: July 17, 2018

Revised: October 5, 2018

Published: October 31, 2018 

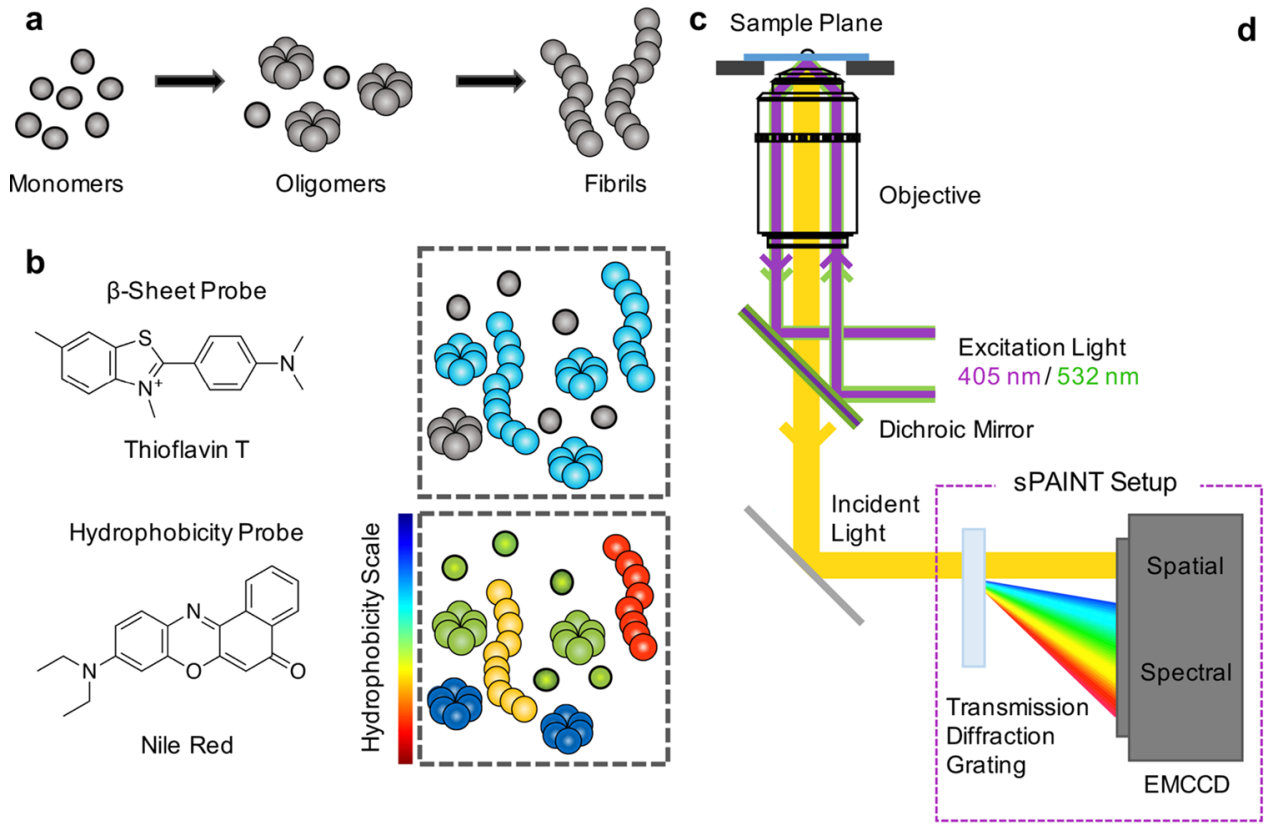

d

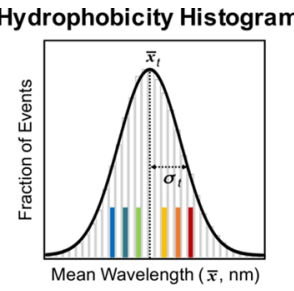

Hydrophobicity Landscape

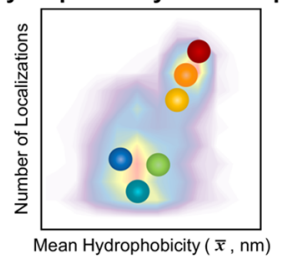

Hydrophobicity Heterogeneity

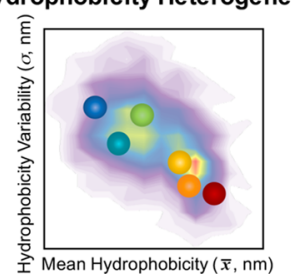

Figure 1. Schematic representation of sPAINT experiment to probe surface hydrophobicity of alpha-synuclein $(\alpha \mathrm{S})$ aggregates. (a) Unlabeled $\alpha \mathrm{S}$ monomers, shown as gray spheres, were incubated under conditions favoring their aggregation into oligomers and ultimately amyloid fibrils. Aliquots were taken, diluted, and imaged using sPAINT. (b) Molecular structure of Thioflavin T (ThT) and Nile Red (NR) dyes used to probe the $\beta$-sheet content and surface hydrophobicity of $\alpha \mathrm{S}$ aggregates, respectively. The fluorescence quantum yield of ThT increases upon binding to the $\beta$ sheet structure of aggregates (represented by the blue colored aggregates). $\alpha \mathrm{S}$ oligomers or fibrils were then probed for hydrophobicity and falsecolored by NR. (c) Schematic of the setup used for the single-molecule sPAINT experiments; single-molecule fluorescence is collected by a high numerical aperture objective lens and passed through a blazed transmission diffraction grating. The fluorescence emission is divided into the spatial region (0th diffraction order) and the spectral region (1st diffraction order) in the image plane and recorded by an EMCCD camera. 405 and 532 $\mathrm{nm}$ lasers are used to sequentially excite the dyes ThT and NR, respectively. (d) sPAINT data terminology: (Upper) Hydrophobicity histograms, cumulative frequency histograms of mean wavelengths of individual $\alpha \mathrm{S}$ aggregates. The histogram is fitted to a Gaussian function and then the total mean hydrophobicity value $\left(\bar{x}_{t}\right)$ and the total standard deviation $(\sigma t)$ are determined. (Middle) Hydrophobicity landscape, density plot of the mean wavelength $(\bar{x})$ and number of localizations $(n)$ of individual $\alpha \mathrm{S}$ aggregates. The value $(\bar{x}, n)$ of each colored-point corresponds to the colored event in the hydrophobicity histogram. (Bottom) Hydrophobicity heterogeneity, density plot of the mean wavelength $(\bar{x})$ and hydrophobicity variability $(\sigma)$ of individual $\alpha \mathrm{S}$ aggregates. The value $(\bar{x}, \sigma)$ of each colored-point corresponds to the colored event in the hydrophobicity histogram.

imaging method, simultaneously recording the spatial position and fluorescence emission spectrum of the solvatochromic dye, Nile Red (NR), ${ }^{15}$ which nonspecifically and transiently binds to protein aggregates. ${ }^{16}$ This method, termed spectrally resolved PAINT (Points Accumulation for Imaging in Nanoscale Topography) or sPAINT, ${ }^{17}$ allows for simultaneous measurement of the morphology/size and relative surface hydrophobicity of individual protein aggregates. We have previously demonstrated that sPAINT can detect hydrophobic differences in a variety of protein aggregates with different morphologies. ${ }^{17}$

Here, we use sPAINT imaging with NR in combination with imaging the benzothiazole derivative Thioflavin $\mathrm{T}$ (ThT), a fluorescent dye that binds to the cross- $\beta$ feature of amyloid structures, ${ }^{1,18}$ to individually characterize the protein aggregates formed during the aggregation of $\alpha \mathrm{S}$ (Figure $1 \mathrm{a}-\mathrm{c}$ ) and follow the changes in surface hydrophobicity. By observing NR-bound $\alpha \mathrm{S}$ aggregates, this approach allows us to image and measure the surface hydrophobicity of the individual protein aggregates formed during an aggregation reaction even before they become ThT active. NR is an uncharged amphiphilic oxazine whose fluorescence absorption and emission spectra are, broadly, sensitive to the local polarity but there is also a contribution from local hydrogen and $\pi$-bonding to the observed spectral shift. The exact mechanism that gives rise to the spectral shift is still being studied. ${ }^{19,20}$ The quantum yield of the NR dye increases with hydrophobicity and previous experiments ${ }^{17}$ on model liposomes with different hydrophobicities showed that this altered the localization probability but not the observed spectral distribution. In this study, we have used NR to super-resolve the relative changes in surface hydrophobicity when aggregates of $\alpha \mathrm{S}$ increase in size from $\sim 30 \mathrm{~nm}$ to $>1 \mu \mathrm{m}$ long fibrils during an aggregation reaction. Furthermore, our methods can measure how the peak spectral emission varies from one aggregate to another (Figure 1d) as well as how much the hydrophobicity of a single aggregate varies by measuring the width of the distribution of the individual sPAINT localizations, thereby determining the range of hydrophobic states available not only between single aggregates but within them as well.

Results. SPAINT Can Follow Changes in the Hydrophobicity of Individual Aggregates During aS Aggregation. We carried out an aggregation reaction of unlabeled $70 \mu \mathrm{M} \alpha \mathrm{S}$ protein incubated at $37{ }^{\circ} \mathrm{C}$ with agitation at $200 \mathrm{rpm}$ and extracted aliquots at different time-points $(1,3,9,24$, and 48 h) for imaging using ThT and sPAINT. Figure $2 \mathrm{a}$ shows representative sPAINT hydrophobicity images of the $\alpha \mathrm{S}$ aggregates at different times. The morphology of the 


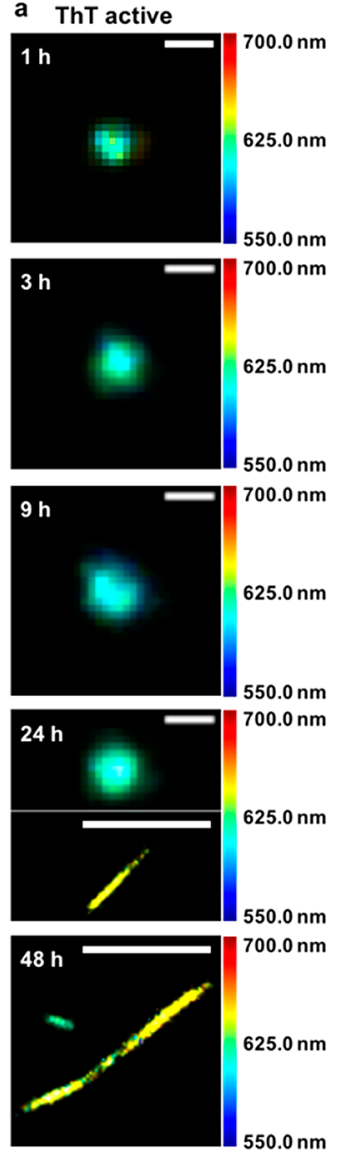

Hydrophobicity Map
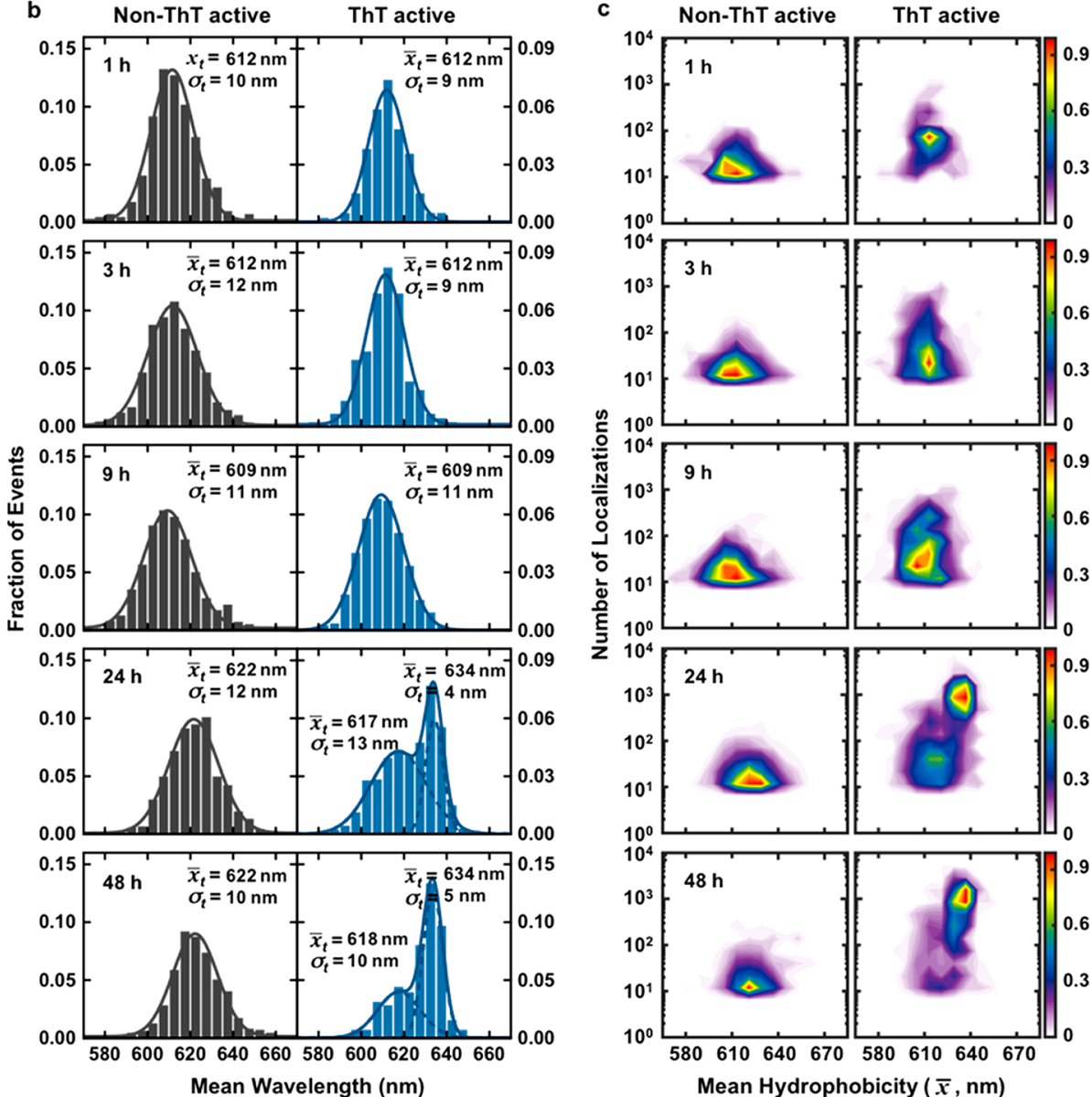

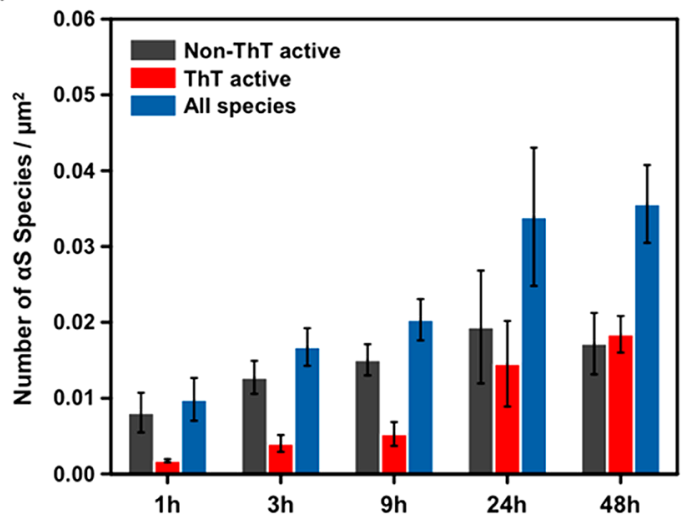

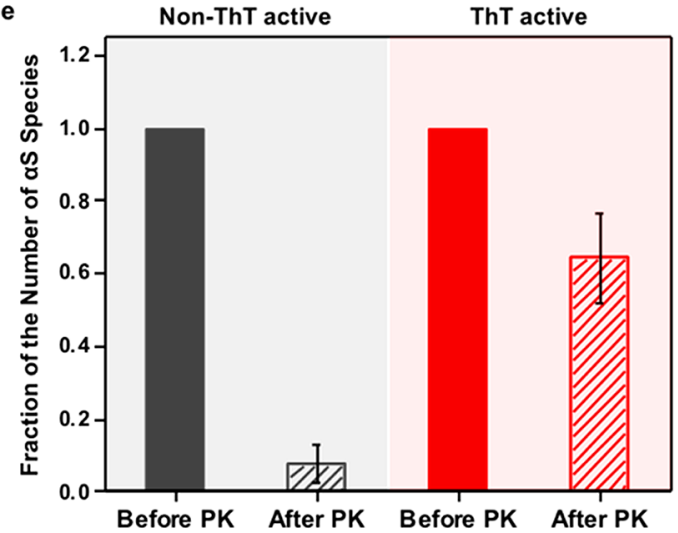

Figure 2. Spectral analysis of $70 \mu \mathrm{M} \alpha \mathrm{S}$ aggregation by sPAINT. (a) Representative sPAINT hydrophobicity images of single ThT active $\alpha \mathrm{S}$ aggregates at different time-points $(1 \mathrm{~h}, 3 \mathrm{~h}, 9 \mathrm{~h}, 24 \mathrm{~h}$, and $48 \mathrm{~h})$ with a hydrophobicity scale. Scale bar is $100 \mathrm{~nm}$ in $1,3,9$, and upper $24 \mathrm{~h}$, and $1 \mu \mathrm{m}$ in bottom 24 and $48 \mathrm{~h}$. (b) Total hydrophobicity populations of non- ThT active and ThT active $\alpha \mathrm{S}$ species $\left(1 \mathrm{~h}, \mathrm{~N}_{(\mathrm{non}-\mathrm{ThT})}=551, N_{(\mathrm{ThT})}=119 ; 3\right.$ h, $N_{(\text {non-ThT) }}=1,091, N_{(\mathrm{ThT})}=334 ; 9 \mathrm{~h}, N_{(\text {non-ThT) }}=1,143, N_{(\mathrm{ThT})}=401 ; 24 \mathrm{~h}, N_{(\text {non-ThT) }}=1,094, N_{(\mathrm{ThT})}=820 ; 48 \mathrm{~h}, N_{(\text {non-ThT) }}=502, N_{(\mathrm{ThT})}=$ 538). (c) Hydrophobicity landscapes of individual aggregates at the different time-points with a density scale. (Lookup table: population density) (d) The number of $\alpha \mathrm{S}$ species per $\mu \mathrm{m}^{2}$ as a function of time. These data correspond to the mean and standard deviation of five independent experiments. (e) PK digestion assays of a 2 day $\alpha \mathrm{S}$ aggregation. The fraction of the number of $\alpha \mathrm{S}$ species in the PK-exposed sample (1 h) was divided by the number of $\alpha \mathrm{S}$ species in the initial sample (total number of $\alpha \mathrm{S}$ species: $N_{\text {(non-ThT) }}=90, N_{(\text {ThT) }}=185$ ). These data correspond to the mean and standard deviation of three independent experiments.

aggregates changed from globular/spherical particles (hereby defined as "oligomeric" species) to elongated species (hereby defined as "fibrillar" species). In parallel to these morphological changes, the emission spectra of the aggregates shifted to longer wavelengths (represented as a shift in color of the hydrophobicity images from green to yellow). This suggests that the aggregates at the late stages, the fibrillar species, have less solvent accessible hydrophobic patches than early stage aggregates. To compare the hydrophobicity of aggregates as a function of time, we constructed the histograms of hydrophobicity by extracting the mean wavelength for each aggregate at every time-point (Figure $2 b$ ). The non-ThT 


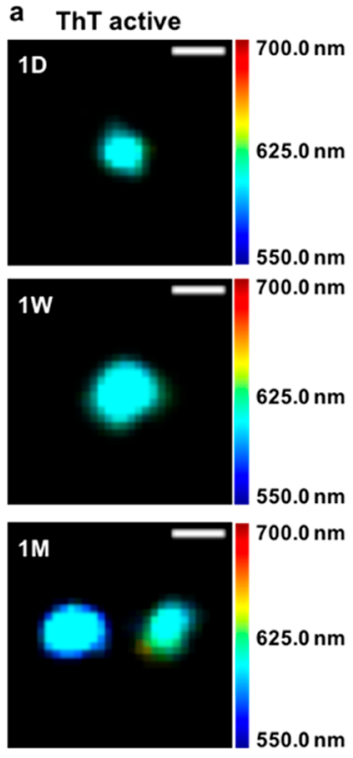

Hydrophobicity Map

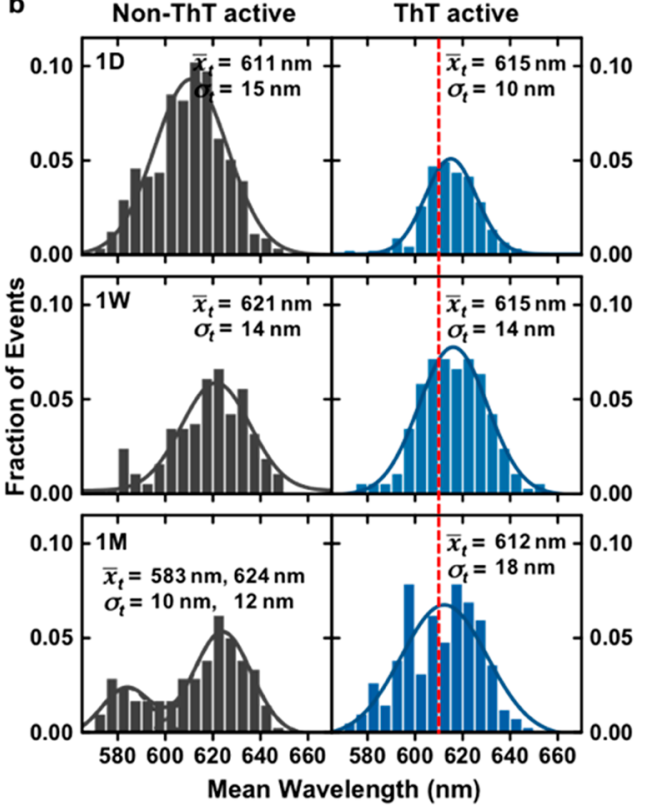

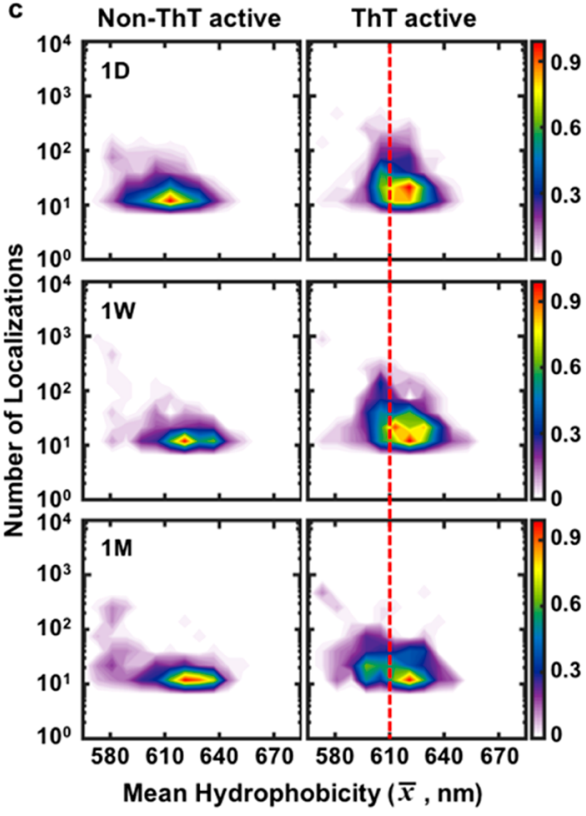

f
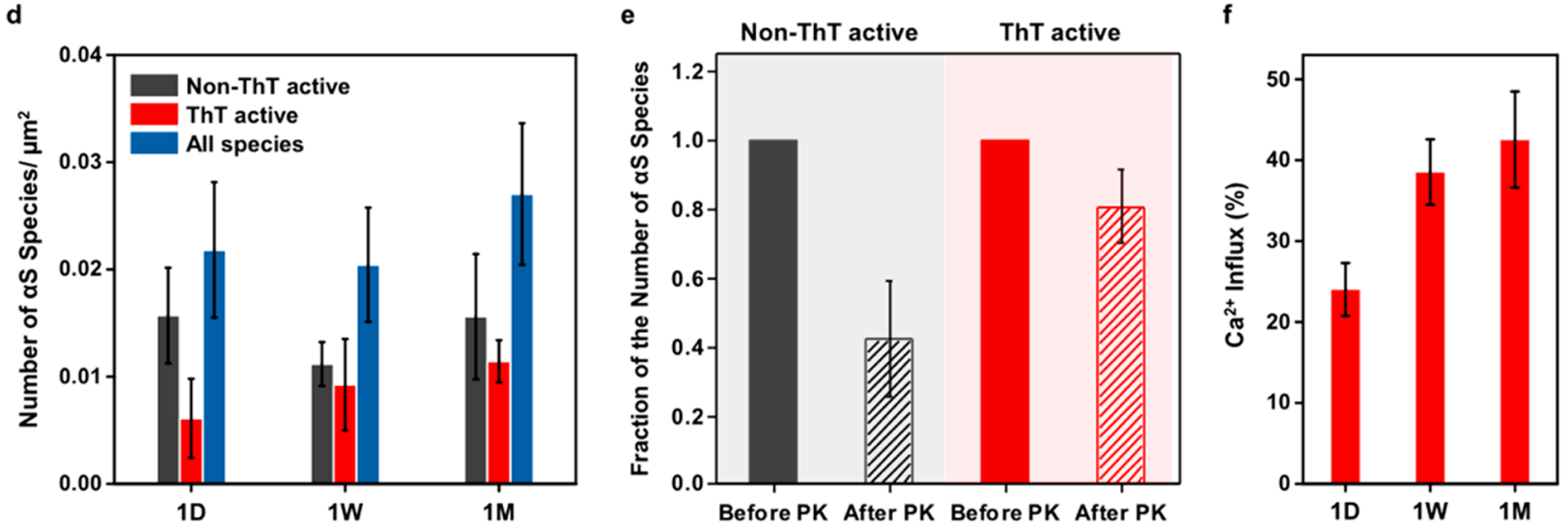

Figure 3. Spectral analysis of $1 \mu \mathrm{M} \alpha \mathrm{S}$ aggregation by sPAINT. (a) Representative sPAINT hydrophobicity image of single ThT active $\alpha \mathrm{S}$ aggregates at different time-points (1day (1D), 1 week (1W), and 1 month (1M)) with a hydrophobicity scale. Scale bar is $100 \mathrm{~nm}$. (b) Total hydrophobicity populations of non-ThT active and ThT active $\alpha \mathrm{S}$ species $\left(1 \mathrm{D}, \mathrm{N}_{(\mathrm{non}-\mathrm{ThT})}=642, N_{(\mathrm{ThT})}=250 ; 1 \mathrm{~W}, N_{(\text {non-ThT) }}=207, N_{(\mathrm{ThT})}=171\right.$; $1 \mathrm{M}, N_{\text {(non-ThT) }}=178, N_{(\mathrm{ThT})}=243$. (c) Hydrophobicity landscapes of individual aggregates at the different time-points with a density scale. (Lookup table: population density.) (d) Number of $\alpha \mathrm{S}$ species $/ \mu \mathrm{m}^{2}$ as a function of time. These data correspond to the mean and standard deviation of three independent experiments. (e) PK digestion assays with sPAINT of a 1 week $\alpha \mathrm{S}$ aggregation. The fraction of the number of $\alpha \mathrm{S}$ species in the PK-exposed sample $(1 \mathrm{~h})$ was divided by the number of $\alpha \mathrm{S}$ species in the initial sample (Total number of $\alpha \mathrm{S}$ species: $N_{(\text {non-ThT) }}=65$, $N_{(\mathrm{ThT})}=183$ ). These data correspond to the mean and standard deviation of three independent experiments. (f) $\mathrm{Ca}^{2+}$ influx induced by aggregation mixture of $1 \mu \mathrm{M} \alpha \mathrm{S}$ proteins at the different time-points (1D, 1W, and $1 \mathrm{M})$. The error bars represent the standard deviation from more than 1000 aggregates of one independent experiment.

active species show a small shift in the peak maxima of $\sim 10 \mathrm{~nm}$ with time. This size of this shift is comparable to the standard deviations of the histograms, whereas there is a larger shift in the maximum hydrophobicity by around $20 \mathrm{~nm}$ for the ThT active species over $24 \mathrm{~h}$, to less hydrophobic fibrillar species. This observation clearly suggests that the aggregate surface changes with time. To gain further information on the hydrophobicity of the aggregates, we created density-plots, referred to as hydrophobicity landscapes in Figure 2c, in which the mean wavelength $(\bar{x})$ was plotted on the $X$-axis and the number of localizations $(n)$ on the $Y$-axis. The values of $\bar{x}$ and $n$ were determined by all the individual sPAINT localizations of individual $\alpha \mathrm{S}$ aggregates. The mean wavelength $(\bar{x})$ represents the mean hydrophobicity of single aggregates. For the non-ThT active species in Figure $2 c$, there was no clear change in the hydrophobicity landscape with time, representing a broad population. In contrast, the hydrophobicity landscape of ThT active aggregates showed a clear change and a shift in aggregate population after $24 \mathrm{~h}$. These plots suggest that the species at the early stages of aggregation, oligomeric species, are more hydrophobic, and structurally distinct from fibrils.

$\alpha S$ Aggregates at Lower Concentration Undergo Slow Changes in Surface Hydrophobicity. Next we incubated $\alpha \mathrm{S}$ at a low monomer concentration $(1 \mu \mathrm{M})$ at $37{ }^{\circ} \mathrm{C}$, closer to physiological conditions, over a month (1 day, 1 week, and 1 month). We then imaged the aggregates formed using sPAINT. Surprisingly, we observed slow surface structural changes over this period of time (Figure 3a), with the formation of more hydrophobic aggregates in both non-ThT 
and ThT active species (Figure $3 \mathrm{~b}$ and $\mathrm{c}$ ). This result suggests that the aggregates undergo slow conformational changes that lead to the formation of more hydrophobic aggregates compared to the aggregation reaction of $70 \mu \mathrm{M} \alpha \mathrm{S}$ (the population to the left of the red line at $610 \mathrm{~nm}$ in Figure $3 \mathrm{~b}$, the mean hydrophobicity of the aggregates at the early stages of $70 \mu \mathrm{M} \alpha \mathrm{S}$ aggregation).

To test if this slow conversion is biologically important, we measured the ability of these aggregates to permeabilize membranes and hence allow the entry of calcium ions $\left(\mathrm{Ca}^{2+}\right)$. To achieve this we used $100 \mathrm{~nm}$ liposomes filled with $\mathrm{Ca}^{2+}$ sensitive dyes that we have recently developed to measure aggregate toxicity. ${ }^{2}$ We tethered thousands of 1-palmitoyl-2oleoyl-sn-glycero-3-phosphocholine (POPC) vesicles (filled with the $\mathrm{Ca}^{2+}$ sensitive dye Cal-520) onto glass coverslips via biotin-neutravidin linkage. The addition of molecules that induce membrane permeability allows the entry of $\mathrm{Ca}^{2+}$ into the vesicles which leads to a change in the localized fluorescence intensity of the dye in the vesicles that can be detected with total internal reflection microscopy (TIRFM). We incubated the vesicles with $\mathrm{Ca}^{2+}$ containing $\mathrm{L} 15$ buffer before we added aggregates formed at different time points (1 day, 1 week, and 1 month) to the vesicles. We observed a significant increase in membrane permeability (toxicity) between aggregates formed after 1 week and 1 month compared to 1 day of $1 \mu \mathrm{M}$ protein aggregation (Figure 3f), which correlates with the increase in the number of ThT active species (the population in the left of red line in Figure $3 \mathrm{~b}$ ). This suggests that these ThT active species, which still have an increased hydrophobic surface, are predominantly responsible for inducing membrane permeabilization and the formation of these aggregates would need to be inhibited before the slow conversion to prevent increased damage to cells. This result is consistent with a recent study of two populations of enriched oligomers which identified that membrane disruption was caused a highly lipophilic element, promoting strong membrane interactions and a structured region that inserts into lipid bilayers and disrupts their integrity. ${ }^{21}$

The Surface of $\alpha$ S Oligomers is Different from $\alpha$ S Fibrils. To better characterize the aggregates formed, we plotted the hydrophobicity landscape for all the species observed during the aggregation reaction of $70 \mu \mathrm{M} \alpha \mathrm{S}$ proteins (Figure 4a). This plot shows that there is one species (A) that is not ThT active and two species (B and $\mathrm{C}$ ) that are ThT active. The non-ThT active species are formed first, corresponding to the low FRET species observed previously, ${ }^{1,14}$ and do not change in morphology during the aggregation; they are very diverse in hydrophobicity and number of localizations, suggesting they exist in a range of different structures. The ThT active species become prominent after $9 \mathrm{~h}$, corresponding to the high FRET, more toxic oligomers we previously observed. The ratio of ThT active to non-ThT active increases from $18 \%$ to $50 \%$ as the reaction proceeds in good agreement with our kinetic model. ${ }^{14}$ After $24 \mathrm{~h}$, the ThT active species are less hydrophobic and less diverse in surface structure (Figure $2 \mathrm{~b}$; $1 \mathrm{~h}, 3 \mathrm{~h}$, and $9 \mathrm{~h}, \bar{x}_{t}=\sim 610 \pm 0.6 \mathrm{~nm}, \sigma_{t}=\sim 10 \pm 0.5 \mathrm{~nm} ; 24$ and $48 \mathrm{~h} \bar{x}_{t}=\sim 635 \pm 0.5 \mathrm{~nm}, \sigma_{t}=\sim 5 \pm 0.4 \mathrm{~nm}$ ) corresponding to high FRET fibril-like oligomers and fibrils. Overall, these data qualitatively agree very well with our previous kinetic model, ${ }^{14}$ although a direct quantitative comparison was not possible. The population of aggregates on the surface does not directly correlate to that in solution due to differences in surface binding as previously observed

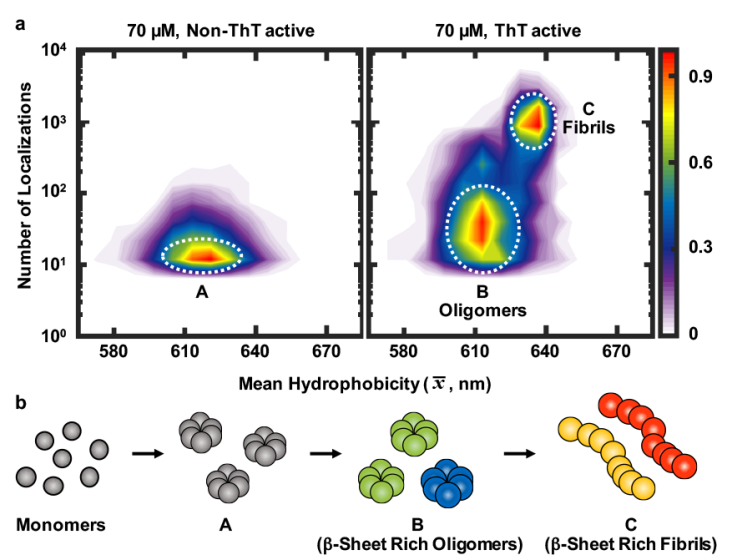

Figure 4. Probing hydrophobicity changes between non-ThT active and ThT active $\alpha \mathrm{S}$ species (a) Hydrophobicity landscapes of nonThT active and ThT active species from all time points for the $70 \mu \mathrm{M}$ $\alpha \mathrm{S}$ aggregation. (Lookup table: population density.) (b) Cartoon showing $\alpha$ S protein aggregation; each $\alpha \mathrm{S}$ species in (A), (B), and (C) corresponds to the $\alpha \mathrm{S}$ species indicated by the area in a white circle area in (a). (A) Non-ThT active small oligomers, (B) ThT active, $\beta$ sheet rich larger oligomers, and (C) ThT active, $\beta$-sheet rich fibrils.

with $\mathrm{A} \beta .^{22}$ However, the qualitative agreement shows that all the main oligomeric species that are formed in solution also bind to the surface and are imaged in our experiment. In contrast to the types of aggregates observed during the aggregation reaction of $70 \mu \mathrm{M} \alpha \mathrm{S}$, there seem to be only two species present when we incubated $\alpha \mathrm{S}$ at $1 \mu \mathrm{M}$ concentration (Figure 3c). The high FRET fibril-like oligomers were absent, suggesting that these are formed from the more hydrophobic ThT active oligomers at higher protein concentrations. Interestingly, the species formed at $1 \mu \mathrm{M}$ concentration showed a wider range of variation in surface hydrophobicity, including more hydrophobic species, than those formed during an aggregation reaction using $70 \mu \mathrm{M} \alpha \mathrm{S}$ proteins.

Size Analysis and Proteinase K Digestion Assay Identify Structural Differences between Non-ThT Active and ThT Active $\alpha S$ Species. We also examined the size of the species formed during these experiments by directly measuring the aggregate size from the super-resolution fluorescence images with an experimentally determined localization precision of around $10 \mathrm{~nm}$ at typical detected photon numbers $(\sim 700$ photons, Supporting Information Figure 1). In particular, we measured the size of diffraction-limited aggregates, which could correspond to the oligomeric species (Supporting Information Figures 2 and 3) by calculating a full width halfmaximum (fwhm) from the standard deviation of a twodimensional Gaussian fit. Aggregates with a wide range of sizes are formed and the non-ThT active species were on average smaller than the ThT active species, $60 \pm 2.6$ and $80 \pm 5.5 \mathrm{~nm}$, for non-ThT and ThT active species, respectively ( $p=0.05$ by $t$ test), which is consistent with the model that the non-ThT active species convert and grow into the ThT active species.

To correlate our measurements of hydrophobicity with aggregate structure, we determined the PK resistance of the aggregates formed after a 2 day incubation of $70 \mu \mathrm{M} \alpha \mathrm{S}$ proteins, which contains a mixture of all aggregate surface structures (Figure 2e). We imaged the aggregates on a glass coverslip surface and exposed the aggregates to PK for $1 \mathrm{~h}$ at $37^{\circ} \mathrm{C}$. We then reimaged the same field of view and measured the fraction of fluorescent puncta that remained detectable. We found that the non-ThT species were most PK sensitive (85\% 

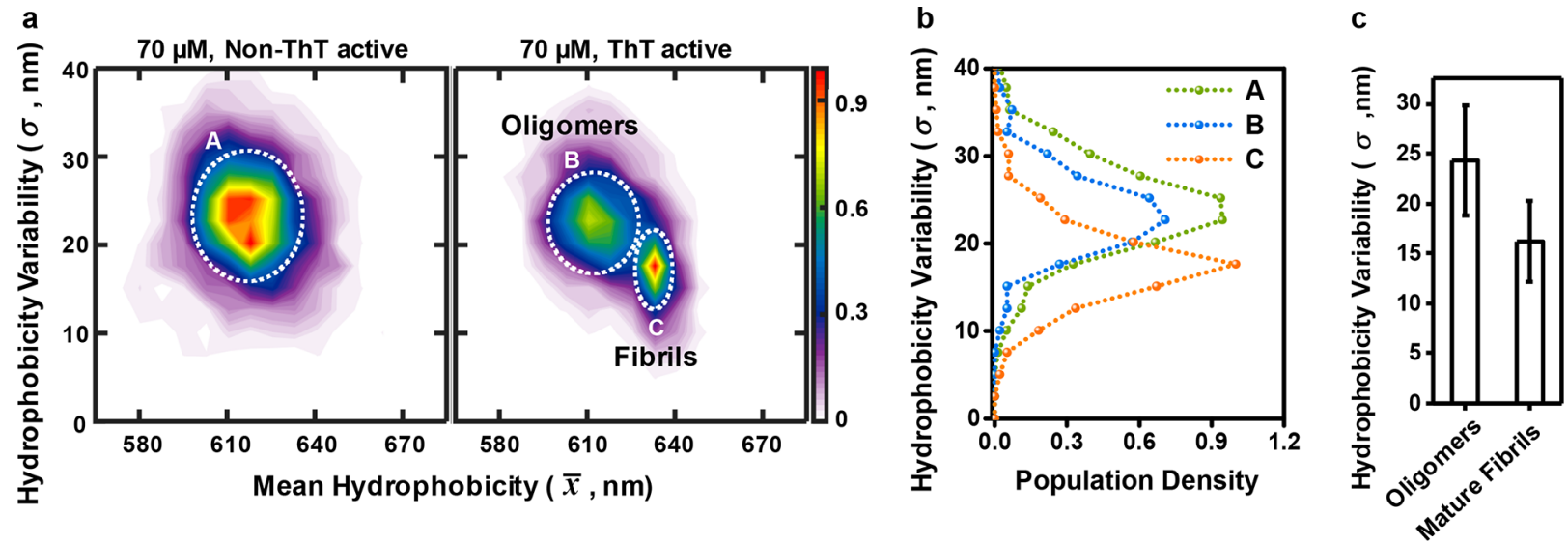

Figure 5. (a) Hydrophobicity heterogeneity (mean hydrophobicity versus hydrophobicity variability) of non-ThT active and ThT active species from all time points for the $70 \mu \mathrm{M} \alpha \mathrm{S}$ aggregation. (Lookup table: population density). (b) Histogram of hydrophobicity variability at $610 \mathrm{~nm}$ through the center of (A) and (B) and at $633 \mathrm{~nm}$ through the center of (C) using the data in Figure 5a. (c) Mean and standard deviation (error bars) of the hydrophobicity variability of oligomeric species at early stages (non-ThT active and ThT active species) and ThT active mature fibrillar species at later-stages with a mean hydrophobicity over $630 \mathrm{~nm}$ and ellipticity less than 0.2 . The measured width of oligomeric species is larger than that of mature fibrillar species $(p<0.0001$ by $t$ test).

of aggregates were degraded), as might be expected due to lack of detectable $\beta$-sheet regions. Interestingly, the aggregates formed at $1 \mu \mathrm{M}$ concentration are slightly less $\mathrm{PK}$ sensitive compared to the aggregates at $70 \mu \mathrm{M}$ concentration (Figure $3 \mathrm{e})$, which is consistent with our observation of the formation of more hydrophobic aggregates, which could have more $\beta$ sheet structures, in both non-ThT and ThT active species at 1 $\mu \mathrm{M}$. These data show that the different aggregates detected by sPAINT also have different structural properties.

The Surface of $\alpha S$ Oligomers Varies in Hydrophobicity More than $\alpha S$ Mature Fibrils. We also measured the distribution of sPAINT spectral shifts observed from individual aggregates. Control experiments showed that the measured width of the distribution of sPAINT spectral shifts of $\alpha \mathrm{S}$ aggregates was due to the variation in the hydrophobicity of the individual aggregates, not the measurement ${ }^{17}$ (Supporting Information Figures 4-6). Using the measured standard deviation of the spectral positions of individual $\alpha \mathrm{S}$ aggregates, we plotted the hydrophobicity heterogeneity, mean hydrophobicity versus hydrophobicity variability, for all the species observed during the aggregation reaction of $70 \mu \mathrm{M} \alpha \mathrm{S}$ proteins (Figure $5 \mathrm{a}$ and $\mathrm{b}$ ). There are three distinct $\alpha \mathrm{S}$ species present, in agreement with the results shown in Figure 4. We then compared the heterogeneity of individual oligomers and mature fibrils. We defined mature fibrils as ThT active aggregates with a mean wavelength over $630 \mathrm{~nm}$, and ellipticity less than 0.2 because these species does not appear in nonThT active species and at the early stage of the aggregation (Supporting Information Figure 3). We have also measured the hydrophobicity of mature fibrils formed in the aggregation of $70 \mu \mathrm{M} \alpha \mathrm{S}$ proteins after $168 \mathrm{~h}$ (Supporting Information Figure 7). The total mean hydrophobicity and standard deviation of the mature fibrils of $168 \mathrm{~h}$ are around 633 and $4 \mathrm{~nm}$, respectively (Supporting Information Figure 7a), and show a clearly well-defined feature in the hydrophobicity landscape and hydrophobicity heterogeneity (Supporting Information Figure $7 b$ and $c$ ). This feature matches that observed at the later stages of $70 \mu \mathrm{M} \alpha \mathrm{S}$ protein aggregation, confirming that it is due to fibril formation. We found that on average individual oligomers have a significantly larger spectral width than individual fibrils, and the variation in these widths for oligomers is also larger than fibrils (Figure $5 c, p<0.0001$ by $t$ test). To determine the distribution of wavelength values one would expect from NR in a well-defined environment within our imaging system, we performed a (photon matched) control experiment using $\beta$-lactoglobulin $(\beta \mathrm{LG})$, a wellstructured protein. Any distribution whose width is larger than that measured for $\beta \mathrm{LG}$ arises from the heterogeneity in the structure of the protein aggregates, and not the instrumentation. Previous studies using $\beta \mathrm{LG}$, which exists as a dimer at room temperature and neutral $\mathrm{pH}$ and binds to $\mathrm{NR},{ }^{15}$ have shown that the peak maximum of the emission spectrum of NR-bound $\beta \mathrm{LG}$ at the ensemble level is around $600 \mathrm{~nm}$, which is consistent with our sPAINT results of $\beta \mathrm{LG}$ (Supporting Information Figure 8). We observed a narrower spectral width of $\beta \mathrm{LG}$ ( $6 \pm 0.6 \mathrm{~nm}$, Supporting Information Figure 8a) than for the $\alpha \mathrm{S}$ aggregates (more than $10 \pm 0.5 \mathrm{~nm}$ for the aggregates at the early stages and bimodal distributions for the aggregates at the later stages in Figures $2 b$ and $3 b$ ). This indicates that the broader spectral population of $\alpha \mathrm{S}$ aggregates, in particular with the aggregates at the early stages, is due to the variations of the individual aggregate surfaces, not the lower number of NR localizations with the aggregates at the early stages or a technical artifact/limitation of the instrumentation. Overall, this result shows that individual oligomers have a larger variation in their surface hydrophobicity and hence surface structure than fibrils, and also that their average surface structure is more diverse, as reflected in the hydrophobicity landscapes.

Discussion. The changes observed in the surface hydrophobicity during aggregation suggest intriguing connections between the underlying structural transformations and selforganization phenomena previously characterized for atomic and molecular clusters, protein folding, and crystallization. ${ }^{23}$ Systems that exhibit efficient relaxation to a well-defined structure are characterized by potential energy landscapes organized so that relaxation is guided toward the global minimum. ${ }^{24-26}$ Self-organization occurs when there is a welldefined global potential energy minimum, connected to higher local minima by low downhill energy barriers. ${ }^{23}$ The landscape is then "unfrustrated", ${ }^{27,28}$ since there are no competing low energy morphologies separated from the target structure by 
high barriers. Although it is important not to overinterpret the changes in hydrophobicity, it is instructive to formulate a prediction for the structure of the underlying energy landscape that is consistent with the present results.

Relaxation on a funnelled potential energy landscape is driven by lowering the potential energy at the expense of reducing the entropy. There is a range of temperature where this first orderlike transition corresponds to a well-defined, kinetically accessible, free energy minimum. The systematic changes observed for the ThT active population in terms of the $\bar{x}$ and $n$ of the hydrophobicity probe are suggestive of an underlying energy landscape with funneling properties that leads to more compact $\beta$-sheet structures and less variation in surface hydrophobicity. For an aggregate of given size, we would expect conformations with better packing to have lower potential energy. We would also expect the number of configurations compatible with more compact structures to diminish as the structure becomes more ordered, which corresponds to a reduction in the landscape entropy. ${ }^{29}$ The time scales involved for the ThT active aggregates to achieve a more uniform hydrophobicity are compatible with the rates calculated for rearrangements of amyloidogenic peptide dimers. In particular, for the amyloidogenic heptapeptide GNNQQNY several alternative dimer arrangements are predicted to form rapidly, probably compatible with a "dock and lock" mechanism. ${ }^{30-32}$ However, the interconversion paths that lead to the cross $\beta$-sheet structure correspond to time scales of hours to days at $300 \mathrm{~K}^{33}$ The present results suggest that the energy landscape for an $\alpha \mathrm{S}$ aggregate has funneling properties leading to a relatively homogeneous family of low-energy ThT active structures. Relaxation would be guided by an energetic driving force, subject to downhill barriers that correspond to time scales in the order of hours to days, and the energy landscape may be similar for other aggregating proteins, given that they form structurally similar fibrils. This observation suggests that initially there is a range of structurally diverse oligomers with different surfaces, and that in the case of $\alpha \mathrm{S}$ they are more hydrophobic than at later timepoints. In contrast aggregates formed at later time points are significantly less structurally diverse. Furthermore, when aggregation occurs at lower protein concentration, relaxation is slower, and hence a wider range of oligomer structures and properties can be formed if left for longer times. These aggregates may be harder to remove or more toxic, suggesting that it is important to remove aggregates before they become more structurally diverse and failure to do this may contribute to the development of neurodegenerative disease. A funneling energy landscape with a reduction in structural diversity as aggregation proceeds may also be a common feature of other aggregating proteins.

In conclusion, we have measured the surface hydrophobicity of individual $\alpha \mathrm{S}$ protein aggregates, formed during an aggregation reaction, using a multidimensional super-resolution imaging method. We have found that the small soluble $\alpha \mathrm{S}$ oligomers display a higher surface hydrophobicity than mature fibrils and coexist in a wider range of more hydrophobic states. Furthermore, individual oligomers show more variation in surface hydrophobicity than individual fibrils. For the first time, we have determined quantitatively the diversity of the surfaces of $\alpha \mathrm{S}$ protein aggregates at the single-aggregate level, providing insights into how the surface properties and heterogeneity of aggregates change as aggregation proceeds.

\section{ASSOCIATED CONTENT}

\section{Supporting Information}

The Supporting Information is available free of charge on the ACS Publications website at DOI: 10.1021/acs.nanolett.8b02916.

Experimental methods; spatial and spectral localization precision of the sPAINT instrument after fiducial correction; size analysis of $\alpha \mathrm{S}$ oligomers; mean hydrophobicity and ellipticity as a function of the size of all small aggregates; hydrophobicity variability distribution of aggregates as a function of the number of localizations; effect of a number of localizations on wavelength; mean of the standard deviation (hydrophobicity variability) of oligomeric and fibrillar species at different number of localizations; sPAINT experiments with mature fibrils of $70 \mu \mathrm{M} \alpha \mathrm{S}$ proteins; technical control sPAINT experiments with $\beta \mathrm{LG}$ proteins (PDF)

\section{AUTHOR INFORMATION}

\section{Corresponding Authors}

*E-mail: dk10012@cam.ac.uk.

*E-mail: s1591@cam.ac.uk.

ORCID

Ji-Eun Lee: 0000-0001-5954-2638

Jason C. Sang: 0000-0002-8567-5415

Patrick Flagmeier: 0000-0002-1204-5340

David J. Wales: 0000-0002-3555-6645

\section{Notes}

The authors declare no competing financial interest.

\section{ACKNOWLEDGMENTS}

C.M.D. is supported by the Wellcome Trust (094425/Z/10/ $\mathrm{Z}$ ) and the UK Biotechnology and Biochemical Sciences Research Council (BB/H003843/1). D.J.W. is supported by the UK Engineering and Physical Science Research Council (EP/L504920/1). S.F.L. is supported by the Royal Society (UF120277). D.K. is a Royal Society Professor of Molecular Medicine and funded by the European Research Council (669237) and MRC (MR/K015850/1). We thank the members of the Klenerman and Lee research groups for their input and discussion; in particular, we would like to thank Dr. Juan Varela, Dr. Aleks Ponjavic, Dr. Marija Iljina, and Dr. Alex Herbert for their helpful discussion.

\section{REFERENCES}

(1) Cremades, N.; Cohen, S. I. A.; Deas, E.; Abramov, A. Y.; Chen, A. Y.; Orte, A.; Sandal, M.; Clarke, R. W.; Dunne, P.; Aprile, F. A.; et al. Direct Observation of the Interconversion of Normal and Toxic Forms of $\alpha$-Synuclein. Cell 2012, 149, 1048-1059.

(2) Flagmeier, P.; De, S.; Wirthensohn, D. C.; Lee, S. F.; Vincke, C.; Muyldermans, S.; Knowles, T. P. J.; Gandhi, S.; Dobson, C. M.; Klenerman, D. Ultrasensitive Measurement of Ca2+ Influx into Lipid Vesicles Induced by Protein Aggregates. Angew. Chem., Int. Ed. 2017, 56, 7750-7754.

(3) Nath, S.; Agholme, L.; Kurudenkandy, F. R.; Granseth, B.; Marcusson, J.; Hallbeck, M. Spreading of Neurodegenerative Pathology via Neuron-to-Neuron Transmission of $\beta$-Amyloid. $J$. Neurosci. 2012, 32, 8767-8777.

(4) Ries, J.; Udayar, V.; Soragni, A.; Hornemann, S.; Nilsson, K. P. R.; Riek, R.; Hock, C.; Ewers, H.; Aguzzi, A. A.; Rajendran, L. 
Superresolution Imaging of Amyloid Fibrils with Binding-Activated Probes. ACS Chem. Neurosci. 2013, 4, 1057-1061.

(5) Pinotsi, D.; Buell, A. K.; Galvagnion, C.; Dobson, C. M.; Kaminski Schierle, G. S.; Kaminski, C. F. Direct Observation of Heterogeneous Amyloid Fibril Growth Kinetics via Two-Color SuperResolution Microscopy. Nano Lett. 2014, 14, 339-345.

(6) Kaminski Schierle, G. S.; Van De Linde, S.; Erdelyi, M.; Esbjörner, E. K.; Klein, T.; Rees, E.; Bertoncini, C. W.; Dobson, C. M.; Sauer, M.; Kaminski, C. F. In Situ Measurements of the Formation and Morphology of Intracellular $\beta$-Amyloid Fibrils by Super-Resolution Fluorescence Imaging. J. Am. Chem. Soc. 2011, 133, 12902-12905.

(7) Roberti, M. H.; Folling, H.; Celej, M. D.; Bossi, M.; Jovin, T. M.; Jares-Erijman, E. A. Imaging Nanometer-Sized $\alpha$-Synuclein Aggregates by Superresolution Fluorescence Localization Microscopy. Biophys. J. 2012, 102, 1598-1607.

(8) Esbjörner, E. K.; Chan, F.; Rees, E.; Erdelyi, M.; Luheshi, L. M.; Bertoncini, C. W.; Kaminski, C. F.; Dobson, C. M.; Kaminski Schierle, G. S. Direct Observations of Amyloid $\beta$ Self-Assembly in Live Cells Provide Insights into Differences in the Kinetics of $\mathrm{A} \beta(1-40)$ and $\mathrm{A} \beta$ (1-42) Aggregation. Chem. Biol. 2014, 21, 732-742.

(9) Zhang, H.; Griggs, A.; Rochet, J. C.; Stanciu, L. A. Vitro Study of $\alpha$-Synuclein Protofibrils by Cryo-EM Suggests a $\mathrm{Cu} 2+$-Dependent Aggregation Pathway. Biophys. J. 2013, 104, 2706-2713.

(10) Chen, S. W.; Drakulic, S.; Deas, E.; Ouberai, M.; Aprile, F. A.; Arranz, R.; Ness, S.; Roodveldt, C.; Guilliams, T.; De-Genst, E. J.; et al. Structural Characterization of Toxic Oligomers That Are Kinetically Trapped during $\alpha$-Synuclein Fibril Formation. Proc. Natl. Acad. Sci. U. S. A. 2015, 112, E1994-E2003.

(11) Wolff, M.; Mittag, J. J.; Herling, T. W.; Genst, E. De; Dobson, C. M.; Knowles, T. P. J.; Braun, D.; Buell, A. K. Quantitative Thermophoretic Study of Disease-Related Protein Aggregates. Sci. Rep. 2016, 6, 1-10.

(12) Campioni, S.; Mannini, B.; Zampagni, M.; Pensalfini, A.; Parrini, C.; Evangelisti, E.; Relini, A.; Stefani, M.; Dobson, C. M.; Cecchi, C.; et al. A Causative Link between the Structure of Aberrant Protein Oligomers and Their Toxicity. Nat. Chem. Biol. 2010, 6, 140147.

(13) Spillantini, M. G.; Schmidt, M. L.; Lee, V. M.-Y.; Trojanowski, J. Q.; Jakes, R.; Goedert, M. $\alpha$-Synuclein in Lewy Bodies. Nature 1997, 388, 839-840.

(14) Iljina, M.; Garcia, G. A.; Horrocks, M. H.; Tosatto, L.; Choi, M. L.; Ganzinger, K. A.; Knowles, T. P. J.; Klenerman, D.; et al. Kinetic Model of the Aggregation of $\alpha$-Synuclein Provides Insights into Prionlike Spreading. Proc. Natl. Acad. Sci. U. S. A. 2016, 113, E1206E1215.

(15) Sackett, D. L.; Wolff, J. Nile Red as a Polarity-Sensitive Fluorescent of Hydrophobic Protein Surfaces. Anal. Biochem. 1987, $167,228-234$.

(16) Sharonov, A.; Hochstrasser, R. M. Wide-Field Subdiffraction Imaging by Accumulated Binding of Diffusing Probes. Proc. Natl. Acad. Sci. U. S. A. 2006, 103, 18911-18916.

(17) Bongiovanni, M. N.; Godet, J.; Horrocks, M. H.; Tosatto, L.; Carr, A. R.; Wirthensohn, D. C.; Ranasinghe, R. T.; Lee, J.-E.; Ponjavic, A.; Fritz, J. V.; et al. Multi-Dimensional Super-Resolution Imaging Enables Surface Hydrophobicity Mapping. Nat. Commun. 2016, 7, 13544.

(18) Cheng, P. N.; Liu, C.; Zhao, M.; Eisenberg, D.; Nowick, J. S. Amyloid $\beta$-Sheet Mimics That Antagonize Protein Aggregation and Reduce Amyloid Toxicity. Nat. Chem. 2012, 4, 927-933.

(19) Ya. Freidzon, A.; Safonov, A. A.; Bagaturyants, A. A.; Alfimov, M. V. Solvatofluorochromism and Twisted Intramolecular ChargeTransfer State of the Nile Red Dye. Int. J. Quantum Chem. 2012, 112, 3059-3067.

(20) Zuehlsdorff, T. J.; Haynes, P. D.; Payne, M. C.; Hine, N. D. M. Predicting Solvatochromic Shifts and Colours of a Solvated Organic Dye: The Example of Nile Red. J. Chem. Phys. 2017, 146, 124504.

(21) Fusco, G.; Chen, S. W.; Williamson, P. T. F.; Cascella, R.; Perni, M.; Jarvis, J. A.; Cecchi, C.; Vendruscolo, M.; Chiti, F.;
Cremades, N.; et al. Structural Basis of Membrane Disruption and Cellular Toxicity by $\alpha$-Synuclein Oligomers. Science 2017, 358, 14401443.

(22) Drews, A.; Flint, J.; Shivji, N.; Jönsson, P.; Wirthensohn, D.; De Genst, E.; Vincke, C.; Muyldermans, S.; Dobson, C.; Klenerman, D. Individual Aggregates of Amyloid Beta Induce Temporary Calcium Influx through the Cell Membrane of Neuronal Cells. Sci. Rep. 2016, 6, 31910.

(23) Wales, D. J. The Energy Landscape as a Unifying Theme in Molecular Science. Philos. Trans. R. Soc., A 2005, 363, 357-377.

(24) Leopold, P. E.; Montal, M.; Onuchic, J. N. Protein Folding Funnels: A Kinetic Approach to the Sequence-Structure Relationship. Proc. Natl. Acad. Sci. U. S. A. 1992, 89, 8721-8725.

(25) Wales, D. J.; Miller, M. A.; Walsh, T. R. Archetypal Energy Landscapes. Nature 1998, 394, 758-760.

(26) Dobson, C. M.; Sali, A.; Karplus, M. Protein Folding: A Perspective from Theory and Experiment. Angew. Chem., Int. Ed. 1998, 37, 868-893.

(27) Bryngelson, J. D.; Onuchic, J. N.; Socci, N. D.; Wolynes, P. G. Funnels, Pathways, and the Energy Landscape of Protein Folding: A Synthesis. Proteins: Struct., Funct., Genet. 1995, 21, 167-195.

(28) Onuchic, J. N.; Luthey-Schulten, Z.; Wolynes, P. G. Theory of Protein Folding: The Energy Landscape Perspective. Annu. Rev. Phys. Chem. 1997, 48, 545-600.

(29) Wales, D. J. Surveying a Complex Potential Energy Landscape: Overcoming Broken Ergodicity Using Basin-Sampling. Chem. Phys. Lett. 2013, 584, 1-9.

(30) Esler, W. P.; Stimson, E. R.; Jennings, J. M.; Vinters, H. V.; Ghilardi, J. R.; Lee, J. P.; Mantyh, P. W.; Maggio, J. E. Alzheimer's Disease Amyloid Propagation by a Template-Dependent Dock-Lock Mechanism. Biochemistry 2000, 39, 6288-6295.

(31) Massi, F.; Straub, J. E. Energy Landscape Theory for Alzheimer's Amyloid $\beta$-Peptide Fibril Elongation. Proteins: Struct. Funct., Genet. 2001, 42, 217-229.

(32) Nguyen, P. H.; Li, M. S.; Stock, G.; Straub, J. E.; Thirumalai, D. Monomer Adds to Preformed Structured Oligomers of A $\beta$-Peptides by a Two-Stage Dock-Lock Mechanism. Proc. Natl. Acad. Sci. U. S. A. 2007, 104, 111-116.

(33) Strodel, B.; Whittleston, C. S.; Wales, D. J. Thermodynamics and Kinetics of Aggregation for the GNNQQNY Peptide. J. Am. Chem. Soc. 2007, 129, 16005-16014. 